\title{
Assessment of the Effectiveness of Health Education for Ibs Women by Examining the Frequency and Disturbance of Gi Symptoms, Quality of Life and Days of Drug Use
}

\author{
Hsiu-Chin Lin, (MSN, RN) \\ National Taipei University of Nursing and Health Sciences/ \\ Mackay Memorial Hospital, Taiwan (R.O.C.) \\ Lee-Ing Tsao, (DNS, RN) \\ National Taipei University of Nursing and Health Sciences, Taiwan (R.O.C.)
}

Doi: 10.19044/esj.2018.v14n12p267 URL:http://dx.doi.org/10.19044/esj.2018.v14n12p267

\begin{abstract}
Background: Irritable bowel syndrome (IBS) is a common and chronic functional disorder, yet few studies have demonstrated the effects of IBS health education. Methods: This study was conducted at the gastrointestinal (GI) clinic of a hospital. A parallel-design control trial for IBS women, in which health education was implemented via individual instruction and the Self-Care Manual for IBS Women, was carried out. The questionnaire utilized in this study covered three areas, namely IBS symptom disturbance and frequency, quality of life, and days of drugs use. The intervention effects were assessed four weeks and eight weeks after the intervention and estimated using the GLMM model (generalized linear mixed model). Results: The experimental and control groups consisted of 31 and 30 participants, respectively. The assessment indicated that health education intervention had significantly reduced symptom frequency in the experimental group after four weeks $(\beta=-2.60, \mathrm{P}<0.01)$ and after eight weeks $(\beta=-3.30, \mathrm{P}<0.01)$; significantly reduced symptom disturbances after four weeks $(\beta=-5.01, \mathrm{P}<$ $0.01)$ and after eight weeks $(\beta=-4.79, \mathrm{P}<0.01)$. Quality of life for both groups increased after eight weeks, with the experimental group experiencing a greater increase than the control group $(\beta=15.20, \mathrm{P}>0.05)$. Drug use decreased by an average of 6.23 days $(\mathrm{P}<0.01)$ and 1.3 days $(\mathrm{P}>0.05)$ in the experimental and control groups, respectively. Conclusions: IBS health education had a positive effect on symptom frequency and disturbance, quality of life and days of drug use.
\end{abstract}

Keywords: Irritable bowel syndrome, symptoms disturbance, quality of life, 
symptom frequency, health education

\section{Introduction}

Irritable bowel syndrome (IBS) is the most common chronic functional gastrointestinal disorder. IBS can be caused by three types of physiological issues, including gastrointestinal motility disorders, hypersensitivity of internal organs to stimuli, and central nervous regulation abnormalities. Eating habits, intestinal motility disorders, enteric nervous system, psychological state, and other factors can lead to IBS. Symptoms occur as the result of abnormal intestinal response to stimuli (food or drinks), enteral infection, or psychological or emotional stress (Drossman, 2006). Studies have indicated regional differences in IBS incidence. For instance, IBS prevalence in Mexico and Latin America is 35.5\% and 17.5\%, respectively. Moreover, in many regions, IBS prevalence among women is higher than that among men (Sperber et al., 2017). According to statistics from 2016, the outpatient rate due to functional disturbances in the digestive system in Taiwan was 4671 among women and 4425 among men (per 100,000 adult population); the rate was substantially higher for women than men (Department of Statistics, Ministry of Health and Welfare, 2016).

Heitkemper, Carter, Ameen, Olden, and Cheng (2002) reported that $53 \%$ of women with IBS perceived limitations with regard to physical activity. On average, women with IBS took leave from work or school for 8.4 days in a year, whereas women without IBS took leave for 4.4 days. Furthermore, women felt that IBS affected the lives of their spouses and children. Japanese scholars indicated that an individual's IBS coping behavior and health perception are correlated with their ability to adapt and psychological health (Sugawara et al., 2017). Relatively few studies have discussed the health behavior of IBS patients in Taiwan. Therefore, this study conducted a pilot research on symptoms and health behavior of women with IBS in a gastroenterology department of a hospital in Taipei City, Taiwan. Among 30 women diagnosed with IBS, 17 did not have a clear understanding of IBS symptoms and matters needing attention in daily life. Moreover, the patients' order of preference with respect to the help that they were hoping to receive was as follows: IBS knowledge, dietary precautions, exercise-induced relief, and stress management.

In view of this, this study aimed to answer the question 'What are the health outcomes of health education for women with IBS?' and develop a selfcare manual containing information about IBS and daily self-care (diet, exercise, and stress management). It is hoped that women with IBS can reduce their symptom frequency, symptom disturbance, and, consequently, drugs use through engagement in healthy behavior, which will in turn lead to lower health insurance costs and an improved quality of life. 


\section{Methods}

\section{Research Design}

The study is a longitudinal and experimental study aimed at examining the effects of health education (which was designed and developed by clinical experts and scholars based on past literature, and involved the use of the SelfCare Manual for IBS Women which covers items such as IBS knowledge, diets and treatments, adaptation to life, and stress management techniques) on women with IBS. To this end, symptom frequency, symptom disturbance, and quality of life were measured at different time points (one month and two months after intervention). Furthermore, the 61 participants in this study were randomly allocated to the experimental (31) and control (30) groups according to their case number, so as to prevent interactional effects among the participants.

Table 1. Research design



O1 (pretest): Measurement of symptom frequency, disturbance level, and quality of life.

O2 (posttest): Symptom frequency, disturbance level, and drugs use after one month of intervention.

O3 (post-posttest): Symptom frequency, disturbance level, drugs use, and quality of life after two months of intervention.

Intervention: The Self-Care Manual for IBS Women was used to provide individual health education.

\section{Conceptual Framework}

In addition to pharmaceutical treatment provided to patients by a gastroenterologist, the experimental group was exposed to health education via the use of the Self-Care Manual for IBS Women. Patients in the control group received general nursing education orally. 




\section{Participants}

This research project received permission from the institutional review board (IRB) of a teaching hospital in Taipei City. The sample was recruited from a gastroenterology department. The sampling criteria included the following: (1) patients with normal mental condition and ability to answer the researcher's questions, complete the questionnaire and record drugs use on their own; (2) patients diagnosed with IBS by a gastroenterologist; and (3) patients willing to participate in the study. Patients with other organic pathologic diseases of the digestive tract were excluded. Random sampling was conducted. Frequency of IBS symptoms was estimated using statistical software (NCSS2000/PASS6.0). The sample size was estimated with the $\chi$ value set to 0.05 , power set to 0.8 and effect size set to 0.3 . In total, 61 patients participated in this study, with 31 patients in the experimental group and 30 patients in the control group.

\section{Research Tools and Research Reliability and Validity}

The research tools included a questionnaire survey and the Self-Care Manual for IBS Women. The questionnaire's content consisted of three parts: (1) demographic information: age, educational level, current working status, income, marital status, living conditions (living alone or with others), etc. Questions were formulated based on literature review. (2) Gastrointestinal symptom frequency and disturbance scales: the scales were developed based on local and international research and in collaboration with a gastroenterologist. Their item content validity CVI was .98. The scales' content reliability was tested on 30 women with IBS prior to the execution of the actual survey. The Cronbach's $\alpha$ of the symptom frequency scale and 
disturbance scale was .66 and .73, respectively, indicating their good reliability. (3) Quality of life scale: The MOS SF-36 (Medical Outcomes Study 36-Item Short Form Health Survey) questionnaire, consisting of 36 items in four levels and eight constructs, was used. The full score for each construct was 100 points, with higher scores indicating better quality of life. The SelfCare Manual for IBS Women was developed based on a literature review, with the use of resources provided by clinical experts (women studies scholars and experts, gastroenterologists, and nutritionists), and based on the IBS Program proposed by Ash and Conkling (2005; translated by Shi). The manual mainly consisted of images, text, and a Q\&A section. Women with IBS were taught to cope with the disease, in order to adapt to changes and improve their quality of life. The manual's content can be divided into the following parts: (1) understanding IBS; (2) introduction to IBS diet; (3) introduction to simple exercises; (4) stress management and relaxation techniques; and (5) reminders for IBS patients. The manual also contained an individual drug use record form and life record card.

\section{Data Analysis}

The English version of SPSS 16.0 for Windows was used. The significance level was set to $\mathrm{P}<0.05$. The study carried out repeated measurements of symptom frequency, symptom disturbance, and quality of life, and utilized the generalized linear mixed model (GLMM) for its analyses. By taking into account the possibility of timing and grouping differences having an effect on symptom frequency, symptom disturbance, and quality of life, corrections were made with respect to the interactional effects between the repeatedly measured variables and the statistical strength of follow-up measurements was increased, which consequently raised the accuracy of the estimations of the effectiveness of health education. Content analysis was employed to perform a subjective evaluation of the effects of the Self-Care Manual for IBS Women that was used in the experimental group.

\section{Results}

\section{Demographic information}

The average age of the patients was $50.68 \pm 15.51$ in the experimental group and $50.30 \pm 16.10$ in the control group; 15 patients were aged 60 years or older $(24.5 \%) .48$ patients had high school (vocational) or college education (78.7\%). 35 participants were unemployed (57.3\%). 21 patients had experienced menopause (34.4\%). The income level was evenly distributed. Most participants were married.

\section{Symptom Frequency and Disturbance Pretest}

The average symptom frequency score per item was $2.04 \pm 0.33$ points 
in the experimental group and 2.07 \pm 0.38 in the control group. Higher scores were obtained by both groups for the following five items (listed in descending order): abdominal distension, abdominal pain relieved by defecation, constipation, abdominal pain, and abdominal colic prior to defecation. The patient symptom frequency values assessed by attending physicians were also examined for the purpose of more objective research. The results indicated that the average item scores in the experimental group and control group were $1.84 \pm 0.40$ and $1.67 \pm 0.24$, respectively. The scores provided by physicians were lower than those that were based on the patients' subjective feeling. With regard to symptom disturbance, the average item scores in the experimental group and control group were $1.94 \pm 0.08$ and $2.03 \pm 0.09$, respectively. Higher scores were obtained by both groups for the same five items, namely, abdominal distension, constipation, abdominal pain, the feeling of inability to leave the bathroom, and abdominal colic prior to defecation.

\section{Quality of Life Pretest}

The average quality of life pretest score in the experimental group was $61.14 \pm 9.52$. The patients scored higher in role limitation due to emotional problems (78.39 \pm 21.13$)$, physical functioning $(78.39 \pm 10.75)$, and vitality $(66.26 \pm 17.50)$. The average score in the control group was $61.43 \pm 7.96$, and higher scores were obtained for physical functioning (78.13 \pm 11.42 分), role limitation due to emotional problems $(71.17 \pm 20.81)$, and physical pain $(66.53 \pm 17.72)$.

The results of the demographic information, symptom frequency, disturbance level, and quality of life pretests in both groups were examined by conducting an independent t test. No statistically significant difference was observed, indicating the homogeneity of the demographic information and dependent variable pretest results for the two groups.

\section{Outcomes in Symptom Frequency, Disturbance Level, and Quality of Life in the Experimental Group and Control Group Symptom Frequency Outcomes after Health Education Intervention}

The effect $\beta$ value of symptom frequency in the pretest was -0.28 $(\mathrm{P}>0.05)$. The predictive $\beta$ value was $-1.36(\mathrm{P}<0.05)$ after one month of intervention and $-1.80(\mathrm{P}<0.01)$ after two months of intervention. An interaction effect was observed between time and grouping. The $\beta$ value in the experimental group was $-2.60(\mathrm{P}<0.01)$ after one month of intervention and $3.30(\mathrm{P}<0.01)$ after two months of intervention. Thus, time affected symptom frequency. Effects were observed after one month and persisted into the second month of intervention (Table 2, Figure 2). 


\section{Symptom disturbance Outcomes after Health Education Intervention}

The effect $\beta$ value of symptom disturbance in the pretest was -1.05 $(\mathrm{P}>0.05)$. The predictive $\beta$ value was $1.23(\mathrm{P}>0.05)$ after one month of intervention and $0.67(\mathrm{P}>0.05)$ after two months of intervention. With regard to the interaction effect between time and grouping, the $\beta$ value in the experimental group was $-5.01(\mathrm{P}<0.01)$ after one month of intervention and $4.79(\mathrm{P}<0.01)$ after two months of intervention. Thus, symptom disturbance was not influenced by time. However, a statistically significant improvement was achieved in the experimental group after one month of intervention and persisted into the second month of intervention (Table 2, Figure 3).

Table 2. Results of GLMM analysis of symptom frequency, disturbance level, and quality of life in the experimental and control groups $(\mathrm{N}=61)$

\begin{tabular}{|c|c|c|c|}
\hline Group Effects & $\bar{\beta}$ & $\mathrm{t}$ & $\mathrm{p}$ \\
\hline \multicolumn{4}{|l|}{ Symptom frequency } \\
\hline Groups: experimental vs. control & -0.28 & -0.21 & 0.83 \\
\hline \multicolumn{4}{|l|}{ Time } \\
\hline Post-posttest vs pretest & -1.80 & -2.52 & $<0.01$ ** \\
\hline Posttest vs pretest & -1.36 & -1.92 & $0.04 *$ \\
\hline \multicolumn{4}{|l|}{ Group*time } \\
\hline posttest $\quad$ Experimental group $*$ post- & -3.30 & -3.29 & $<0.01$ ** \\
\hline Experimental group * & -2.60 & -2.60 & $<0.01$ ** \\
\hline
\end{tabular}

Disturbance level

Groups: experimental vs. control $\quad-1.05 \quad-0.71 \quad 0.48$

Time

$\begin{array}{llll}\text { Post-posttest vs pretest } & 0.67 & 0.80 & 0.43 \\ \text { Posttest vs pretest } & 1.23 & 1.47 & 0.14\end{array}$

Group*time posttest

Experimental group * post- -4.7

Experimental group * posttest $\quad-5.01$

$-4.26$

$<0.01$ * *

Quality of life

Groups: experimental vs. control

21.70

1.23

0.22

Time

Post-posttest vs pretest 
Group*time

Experimental group * postposttest
${ }^{*} \mathrm{P}<0.05 ; \mathrm{P}<0.01{ }^{*}$; posttest indicates one month after intervention; post-posttest indicates two months after intervention

\section{Quality of Life Outcomes after Health Education Intervention}

The effect $\beta$ value of quality of life in the pretest was $21.70(\mathrm{P}>0.05)$. The $\beta$ value was $33.67(\mathrm{P}<0.01)$ after two months of intervention. With regard to the intervention of time and group, the $\beta$ value was $15.20(\mathrm{P}>0.05)$. Thus, quality of life in both groups improved with time. However, the estimated improvement of quality of life in the experimental group was greater than that in the control group (Table 2, Figure 4).

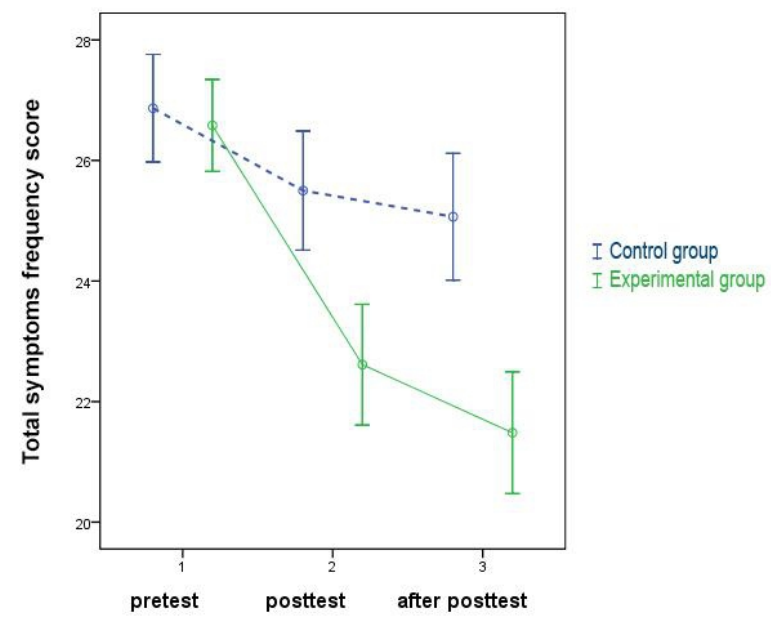

Figure 2. Comparison of symptom frequency outcomes in the experimental and control



Figure 3. Comparison of symptom disturbance outcomes in the experimental and control groups 




Figure 4. Comparison of quality of life outcomes in the experimental and control groups

\section{Differences in Drugs Use Posttest and Post-Posttest Results between the Two Groups}

In this study, the patients' drug use was self-reported. In consideration of the uncertainty of information regarding patients' drugs use prior to research due to the lack of related data, only a t-test was used to compare drug use in the two groups. The average number of days of drugs use in the experimental group was $19.55 \pm 8.46$ days/month in the posttest and $13.32 \pm 10.04$ days/month in the post-posttest, indicating an average decrease of 6.23 days/month $(\mathrm{P}<0.01)$. The average number of days of drug use in the experimental group was $26.97 \pm 2.77$ days/month in the posttest and $25.67 \pm 5.41$ days/month in the post-posttest, indicating an average decrease of 1.3 days/month $(\mathrm{P}>0.05)$.

Statistically significant differences were observed between the experimental group and control group in both the posttest and post-posttest $(\mathrm{P}<0.01)$.

Table 3. Comparison of the average number of days of drugs use $(\mathrm{N}=61)$

\begin{tabular}{|c|c|c|c|c|c|}
\hline & $\begin{array}{l}\text { Experimental group } \\
\text { within-groups paire }\end{array}$ & $\begin{array}{l}(\mathrm{N}=31) \\
\text { ed } t \text {-test }\end{array}$ & $\begin{array}{r}\text { Control group (I } \\
\text { within-groups pair }\end{array}$ & $\begin{array}{l}\mathrm{N}=30) \\
\text { red t-test }\end{array}$ & $\begin{array}{l}\text { Between- } \\
\text { groups }\end{array}$ \\
\hline & $\begin{array}{c}\text { Average } t \\
\text { difference } \\
\text { (days) }\end{array}$ & $\mathrm{P}$ & $\begin{array}{c}\text { Average } t \\
\text { difference } \\
\text { (days) }\end{array}$ & $\mathrm{P}$ & $\begin{array}{c}\text { independent } \\
\text { test } \\
\mathrm{t} \quad \mathrm{P}\end{array}$ \\
\hline Post-posttest - posttest & $-6.23 \pm 5.88$ & $<0.01$ ** & $-1.30 \pm 4.83-1.47$ & 0.15 & $3.57<0.01$ * * \\
\hline
\end{tabular}

\footnotetext{
$\mathrm{P}<0.05^{*} ; \mathrm{P}<0.01^{* *} ;$ posttest indicates one month after intervention; post-posttest indicates two months after intervention
} 
Subjective Evaluation of the Self-Care Manual for IBS Women and Health Education by the Female Participants in the Experimental Group

The content of the opinions expressed by the interviewed female participants was summarized as follows:

(1) Improved understanding of the disease and clarification of misconceptions. Patients said:

"I didn't know that I had IBS and thought that I had cancer;" "The manual does improve my understanding of IBS. It makes you stay on alert and reminds you to change your thinking. The symptoms have also been alleviated."

(2) Learning self-care

A. Exercise and relaxation techniques. $51.6 \%$ of the patients said, "There is no detailed information about IBS in Taiwanese market and online, let alone people who could teach you exercise, deep breathing, and relaxation techniques for distraction. With the illustrated instructions and practice exercises, I can now practice by myself at any time. Discomfort in my stomach decreased greatly."

B. Dietary precautions: 19 patients $(61.2 \%)$ indicated that the manual "provides specific instructions that can be easily understood and followed and helps a patient to understand which foods may cause problems that affect the stomach and intestines."

C. Reduced drugs use and self-monitoring records

24 people (77.4\%) suggested, "The drug and diet record card helps a patient to understand which foods can be eaten again and realize when he or she no longer need drugs because his or her symptoms are gone."

D. Improvement of self-perceived gastrointestinal symptoms

11 participants $(35.4 \%)$ maintained that the most evident improvement occurred with respect to abdominal pain, followed by abdominal distension and diarrhea. Constipation, nausea, and feeling of incomplete evacuation were also alleviated.

E Other

Most of the female participants expressed that, in addition to the manual's portability and the ability to share their knowledge with their families and friends, the manual introduced new topics for discussion. Four participants also experienced the additional benefit of a reduced waist circumference, which was achieved through to abdominal massage.

In sum, the female participants' assessment of the Self-Care Manual for IBS Women demonstrated that the manual improved their understanding about IBS, taught them about ways to relieve gastrointestinal symptoms and enhanced their self-care skills. Furthermore, the participants' drugs use and medical costs were reduced and their quality of life was improved. Thus, a simple, clear, and practical teaching manual should be promoted. 


\section{Discussion and Conclusion}

\section{Discussion and Analysis of Demographic Information}

Past studies indicated that most women with IBS were aged 30-50 years and that disease occurrence decreased with age, which could be related to hormonal factors (Drossman, Camilleri, Mayer, \& Whitehead, 2002). However, 15 participants $(24.6 \%)$ in this study were aged 60 years or older. Among the participants, 21 (34.4\%) had already experienced menopause. These findings did not correspond to those in the study described above. Thus, the presence of other related factors was inferred. A study on the effects of estrogens on patients with IBS reported that estrogens can induce reactions in the intestinal tract, simultaneously promoting complex intestinal irritation and anti-inflammatory effects in the intestines (Mulak, Tache, \& Larauche, 2014). With regard to educational level, most patients had high school or college education (77\%), which was similar to the results of a study by Heitkemper et al. (2002). As proposed by Lin, Wen, and Wei (2007), people with higher education have more opportunities to obtain healthcare information and engage in exercise and behavior beneficial for their health. In this study, 35 participants (57\%) were unemployed. This ratio was higher than that in the study by Silk (2001), in which 329 out of 1204 patients (27.3\%) were unable to work. A related study indicated that as an evident symptom experienced by patients with IBS, abdominal pain has a significant interaction effect on work (Heitkemper et al., 2011). As such, IBS affects work. However, this study did not discuss the taking of leave from work and IBS influence on work. Therefore, it is suggested that future studies conduct more in-depth research on work influence and disturbance in patients with IBS.

\section{Effects of Health Education Intervention on Gastrointestinal Symptoms Symptom Frequency}

The results of the study indicated an improvement in symptom frequency after health education intervention. The results were observed one month after intervention and persisted into the second month after intervention. Earlier studies indicated that spending more time on health education during one outpatient visit effectively reduces the patient's number of outpatient visits. According to Lan and Hung (2000), the objective of health education is to strengthen behavioral changes and self-care abilities through the provision of knowledge in order to reduce injuries, disabilities, and death, increase the living standard and satisfaction and improve quality of life. By implementing health education, hospitals can promote communication between patients and medical personnel, prevent the recurrence of disease and reduce hospital operation costs and the risk of medical disputes. In recent years, the medical market has gradually shifted toward patient-centered care, in which special attention is paid to patients' feelings and the focus is placed 
on meeting patients' needs (Liang, Chen, \& Cheng, 2008). In addition to disease knowledge, the Self-Care Manual for IBS Women developed in this study contained illustrations for dietary guidelines, exercise techniques, and abdominal massage methods. The portability of the manual allows female participants to pay attention to their daily life behavior. Specialist counseling allowed the patients to feel that their privacy was protected, making they feel comfortable about speaking to their physician, which contributed to the decrease in symptom frequency. The results of this study can serve as a basis for the development of IBS care in Taiwan.

\section{Symptom Disturbance Level}

Symptom-induced disturbance in patients improved one month after the health education intervention; this effect was also observed two months after the intervention. Improvements were mainly observed in three types of disturbance, specifically, abdominal distension, constipation, and feeling of incomplete evacuation. These results corresponded to the improvements in symptom frequency. A potential reason for the improvements was that patients' disturbances were mainly caused by symptoms and, thus, a decrease in symptom frequency in the experimental group led a decrease in the disturbance level.

\section{Effects of Health Education Intervention on Quality of Life}

The repeated measurement of patients' quality of life two months after the intervention indicated that quality of life improved with time. This improvement was greater in the experimental group than in the control group $(\beta=15.20)$, which could be explained by the continuous use of the portable manual and counseling service in the experimental group. Patients in the control group only received oral instructions (education communicated orally by nurses) and were not provided with counseling service. As a result, they demonstrated a smaller improvement in symptom frequency and disturbance level as compared to the experimental group, which indirectly affected the improvement in their quality of life. It is suggested that future studies should extend the duration of quality of life monitoring in order to clarify the significance of time effects.

The observed improvements in quality of life after the intervention corresponded to the findings reported by Kennedy et al. (2005) who found that symptoms occurrence and quality of work and social life in IBS patients improved after the implementation of a cognitive behavioral therapy.

\section{Patients' Drugs Use after Health Education Intervention}

In contrast to the control group, a statistically significant difference between post-posttest and posttest results was reached in the experimental 
group. Similarly, Heymann-Mönnikes et al. (2000) reported that the experimental group in their study, which was exposed to different behavioral therapies in combination with drugs use, outperformed the control group, which only received pharmaceutical treatment.

\section{Other Findings}

\section{Differences in Diseases Knowledge between Physicians and Participants}

The IBS symptoms scale in this study was completed by both patients and attending physicians, whose scores differed significantly $(\mathrm{P}<0.05)$. These results corresponded to those reported by Dancey and Backhouse (1993), which indicated that medical personnel often believed that these patients were wasting their physicians' time and, thus, did not formally treat and manage patients' problems. Bengtsson et al. (2007) stated that comprehensive IBS care must include the following: (1) symptoms treatment, (2) confirmation of the patient's issues and needs, (3) confirmation of the diagnosis, and (4) provision of guidance (education) to the patient; moreover, this treatment plan must be followed over a certain period of time. Patients are better able to prepare to symptoms if they are aware of the influencing factors and ways to cope with their symptoms. Patients should be encouraged to act, undertake the responsibility of managing their disease, establish definite feasible goals, and remove environmental factors that can induce their symptoms (Robinson et al., 2006).

\section{Conclusion}

Health education can effectively improve symptom frequency and disturbance level in patients with IBS

Improvement of symptom frequency in the experimental group was significantly higher than that in the control group in both the posttest and postposttest results. In comparison to the control group, patients in the experimental group also experienced a greater improvement in disturbance level with time.

\section{Quality of life improves under the effect of time}

GLMM results indicated time-related improvements in patients' quality of life in both groups. These improvements were greater in the experimental group (6.11 points) than in the control group (4.21 points). Hence, health education with the use of a manual was more beneficial for quality of life than standard oral instructions (education provided orally by nurses).

\section{Health Education Intervention can reduce the days of drugs use}

Patients' drugs use was reduced by the introduction of dieting, 
abdominal exercise examples, revision, and stress management and relaxation techniques in the manual in combination with self-monitoring.

In sum, the female participants in the experimental group maintained that health education improved their knowledge about the disease and clarified previous misconceptions. The manual helped patients to learn about self-care and improve symptoms and disturbance, and introduced topics for discussion with friends, which promoted their interpersonal relationships. Thus, the development and use of the Self-Care Manual for IBS Women brought about actual benefits for women with IBS.

\section{Application and Suggestions}

This study successfully implemented the use of a care manual for patients with IBS. The manual's effects included a reduction in the occurrence of symptoms, disturbances, and number of days of drugs use. In future, this research can be replicated in other medical units. In this study, female participants were provided with a record card for diet and drugs use monitoring. As a result, the female participants engaged in self-care, which increased their attention to their own health, as well as the effectiveness of the study. Most of the participants were unemployed older adults (57\%), which was different from the other overseas studies. However, this study did not explore the relationship between IBS and the inability to work. Thus, future studies can conduct in-depth analyses of IBS age distribution and its effects on life and work.

\section{Limitations}

Due to time limitations, the health effects in this study were monitored for only two months. It is suggested that the monitoring period for future studies on health education intervention can be extended to 6-12 months. After the completion of the study, many participants were willing to continue to receive specialist counseling. For the sake of a more comprehensive IBS care plan, a specialist can be assigned or a hotline can be established to provide a platform for continued education for patients with IBS and other chronic diseases.

\section{References:}

1. Bengtsson, M., Ohlsson, B., \& Ulander, K. (2007). Women With Irritable Bowel Syndrome and Their Perception of a Good Quality of Life. Gastroenterology nursing, 30(2), 74-82. doi:10.1097/01.SGA.0000267924.24180.1a

2. Dancey, C. P., \& Backhouse, S. (1993). Towards a better understanding of patients with irritable bowel syndrome. Journal of Advanced Nursing, 18(9), 1443-1450. doi:10.1046/j.13652648.1993.18091443.x 
3. Department of Statistics, Ministry of Health and Welfare. (2016). 2016 ROC Total Rate of Outpatient Visits (Including Emergency Visits): Number of Outpatients by Disease, Gender, and Age. Retrieved on February 8, 2018, from: https://www.mohw.gov.tw/dl-13446d6ac2b42-ad4f-40ec-bc94- 91d4764a3159.html

4. Drossman, D. A., Camilleri, M., Mayer, E. A., \& Whitehead, W. E. (2002). AGA technical review on irritable bowel syndrome. Gastroenterology, 123(6), 2108-2131. doi:https://doi.org/10.1053/gast.2002.37095

5. Drossman, D. A. (2006). The functional gastrointestinal disorders and the Rome III process. Gastroenterology, 130(5), 1377-1390. doi:10.1053/j.gastro.2006.03.008

6. Heitkemper, M., Carter, E., Ameen, V., Olden, K., \& Cheng, L. (2002). Women With Irritable Bowel Syndrome: Differences in Patients' and Physicians' Perceptions. Gastroenterology nursing : the official journal of the Society of Gastroenterology Nurses and Associates, 25(5), 192-200. doi: 10.1097/00001610-200209000-00004

7. Heitkemper, M., Cain, K. C., Shulman, R., Burr, R., Poppe, A., \& Jarrett, M. (2011). Subtypes of irritable bowel syndrome based on abdominal pain/discomfort severity and bowel pattern. Digestive Diseases and Sciences, 56(7), 2050-2058. doi:10.1007/s10620-0111567-4

8. Heymann-Mönnikes, I., Arnold, R., Florin, I., Herda, C., Melfsen, S., \& Mönnikes, H. (2000). The combination of medical treatment plus multicomponent behavioral therapy is superior to medical treatment alone in the therapy of irritable bowel syndrome. The American Journal of Gastroenterology, 95(4), 981-994. doi:10.1111/j.15720241.2000.01937.x

9. Kennedy, T., Jones, R., Darnley, S., Seed, P., Wessely, S., \& Chalder, T. (2005). Cognitive behaviour therapy in addition to antispasmodic treatment for irritable bowel syndrome in primary care: randomised controlled trial. BMJ, 1-6. doi: 10.1136/bmj.38545.505764.06.

10. Lan, C. F., \& Hung, C. T. (2000). Discussion of the medical expenses payment system. Bimonthly of National Health Insurance, 27, 9-13.

11. Liang, Y. W., Chen, F. J., \& Cheng, Y. L. (2008). Preventable hospitalizations in Taiwan and patients' socioeconomic status. Taiwan Journal of Public Health, 27(1), 81-90. doi:10.6288/tjph2008-27-0108.

12. Lin, Y. C., Wen, C. P., \& Wai, P. M. (2007). Leisure-time physical activity and its association with health behaviors, health status and health-related quality of life among Taiwanese adults. Taiwan Journal of Public Health, 26(3), 218-228. 
13. Mulak, A., Tache, Y., \& Larauche, M. (2014). Sex hormones in the modulation of irritable bowel syndrome. World Journal of Gastroenterology, 20(10), 2433-2448. doi:10.3748/wjg.v20.i10.2433

14. Robinson, A., Lee, V., Kennedy, A., Middleton, L., Rogers, A., \& Thompson, D. G. (2006). A randomized controlled trial of self-help interventions in patients with a primary care diagnosis of irritable bowel syndrome. Gut, 55, 643-648. doi: 10.1136/gut.2004.062901

15. Shi, C. S. (2005). IBS Program: What your doctor may tell you about IBS: Eliminate your symptoms and live a pain-free, drug-free life (Ash, R. N., \& Conkling, W., 2005). Taipei: Yuan Shui Wen Hua.

16. Silk, David B. (2001). Impact of irritable bowel syndrome on personal relationships and working practices. European Journal of Gastroenterology \& Hepatology, 13(11), 1327-1332.

17. Sperber, A. D., Dumitrascu, D., Fukudo, S., Gerson, C., Ghoshal, U. C., Gwee, K. A., . . . Whitehead, W. (2017). The global prevalence of IBS in adults remains elusive due to the heterogeneity of studies: a Rome Foundation working team literature review. Gut, 66(6), 10751082. doi:10.1136/gutjnl-2015-311240

18. Sugawara, N., Sato, K., Takahashi, I., Satake, R., Fukuda, S., Nakaji, S., \& Yasui-Furukori, N. (2017). Depressive Symptoms and Coping Behaviors among Individuals with Irritable Bowel Syndrome in Japan. Internal Medicine, $56(5)$ 493-498. doi:10.2169/internalmedicine.56.7695 